\title{
Blazhko stars: Always distorted?
}

\author{
J. Jurcsik, J. M. Benkő, and B. Szeidl \\ Konkoly Observatory of the Hungarian Academy of Sciences, PO Box 67, 1525 Budapest, Hungary \\ e-mail: jurcsik, szeidl@konkoly.hu \\ Received 25 February 2002 / Accepted 4 April 2002

\begin{abstract}
A reexamination of the amplitude ratios of the photometric and radial velocity changes of Blazhko RRab stars in their different Blazhko phases shows that a normal amplitude ratio occurs near the phase of maximum amplitude. Checking the Fourier parameters of maximum amplitude light curves with different methods proved, however, that none of these light curves
\end{abstract} \\ can be regarded as a normal RR Lyrae type light curve.
}

Key words. stars: variables: RR Lyr - stars: oscillations - techniques: photometric - techniques: radial velocities

\section{Introduction}

Long period (10-100 days) amplitude and/or phase modulation of RRab stars, often called the Blazhko effect, has been known for about a century. Although $30-40 \%$ of the fundamental mode RR Lyrae stars exhibit the phenomenon, theoretical and observational efforts have not led to complete understanding of its physical background. One crucial point that may help in selecting the correct explanation would be to decide what phase of the modulation (if any) corresponds to unmodulated single mode RRab radial pulsation i.e. can be regarded as normal, undistorted behaviour.

It was pointed out by Szeidl (1988) that on the pulsational period - photometric amplitude diagram the large amplitude phases of Blazhko variables fall within the range occupied by normal RRab stars. From the visual inspection of the light curves it can be seen that peculiar shapes occur in the small amplitude phases. These facts indicate that if there is any normal phase of a Blazhko star, it should occur during the phases when the amplitudes are larger. However, we do not know for sure that such a phase really exists, and if it does, whether corresponds to the largest amplitude phase.

In the recent past simple linear relations between the physical properties and the Fourier parameters of the light curves of RRab stars were derived (Kovács \& Walker 1999, and references therein) which have been proven to give similarly accurate results to direct spectroscopic methods. A compatibility criterion $\left(D_{\mathrm{m}}\right)$ of the light curves with those of a large sample of RRab stars was given in Kovács \& Kanbur (1998, see also Jurcsik \& Kovács 1996). This method utilizes the interrelations between the Fourier parameters of the light curve and serves as a firmer restriction on the actual values of the Fourier parameters than their possible ranges. To quantify the

Send offprint requests to: J. M. Benkő,

e-mail: benko@konkoly.hu morphological compatibility between a given RRab light curve and that of a large sample of RRab light curves, the $D_{\mathrm{m}}$ parameter was introduced. This parameter represents the maximum value of the deviations of the observed Fourier amplitudes and phases from their predicted values calculated according to the interrelations. Checking whether these relations give correct values in any phase of the Blazhko cycle can help decide which is the normal phase during the Blazhko modulation.

Another tool for checking similarity (or difference) of a Blazhko star to the archetype of the class is to follow their behaviour in the photometric amplitude $\left(A_{V}\right)$ - radial velocity amplitude $\left(A_{v_{\text {rad }}}\right)$ plane. This type of diagram was first constructed by Kurochkin (1959), who showed that these observed quantities are linearly correlated for different types of pulsating stars. Preston \& Spinrad (1967, hereafter PS67) documented the behaviour of three Blazhko type variables in a similar plot.

In this paper we reexamine the light change variations of Blazhko stars utilizing the $A_{V}-A_{v_{\text {rad }}}$ relation, compare the measured and calculated $[\mathrm{Fe} / \mathrm{H}]$ values (Jurcsik \& Kovács 1996) at different Blazhko phases, and measure the compatibility of the light curves with that of normal RRab stars using the interrelations between the Fourier parameters (Kovács \& Kanbur 1998), in order to answer the question: what is the normal state of a Blazhko star?

\section{2. $A_{v_{\text {rad }}}$ vs. $A_{V}$}

Although very few radial velocity observations of Blazhko variables have been published since the appearance of the PS67 paper, the $A_{V}-A_{v_{\text {rad }}}$ diagram of a more complete sample of RR Lyrae stars given by Liu (1991) provides the opportunity and also makes it necessary to refine the positions of Blazhko variables on this diagram. In order to do so, the literature was searched for radial velocity measurements of known Blazhko variables. 
Table 1. Compilation of radial velocity and photometric amplitudes of Blazhko stars.

\begin{tabular}{|c|c|c|c|c|c|c|c|c|}
\hline \multirow[b]{2}{*}{ Name } & \multicolumn{4}{|c|}{ Radial velocity data } & \multicolumn{4}{|c|}{ Photometric data } \\
\hline & Time interval $^{\mathrm{a}}$ & $A_{v_{\mathrm{rad}}}^{\mathrm{b}}\left[\mathrm{km} \mathrm{s}^{-1}\right]$ & $\Phi_{\mathrm{B}}$ & $\operatorname{Ref}^{\mathrm{c}, \mathrm{d}}$ & Time interval $^{\mathrm{a}}$ & $A_{V}^{\mathrm{b}}[\mathrm{mag}]$ & $\Phi_{\mathrm{B}}$ & Ref. $^{c}$ \\
\hline \multirow[t]{3}{*}{ RS Boo } & $46951-46959$ & $68 \pm 1$ & $0.84 \pm 0.01$ & $2(\mathrm{M})$ & $46948-46949$ & $1.24 \pm 0.01$ & $0.83 \pm 0.001$ & 2 \\
\hline & 46879 & $65 \pm 1$ & $0.69 \pm 0.001$ & $2(\mathrm{M})$ & $38844-38878$ & $1.13 \pm 0.02$ & $0.62 \pm 0.03$ & 1 \\
\hline & $38928-38930$ & $56 \pm 5$ & $0.75 \pm 0.002$ & $13(\mathrm{M})$ & 43716 & $1.18 \pm 0.02$ & 0.75 & 4 \\
\hline \multirow[t]{2}{*}{ XZ Cyg } & $32688-32690$ & $66 \pm 10^{\mathrm{e}}$ & $0.0 \pm 0.02$ & $9(\mathrm{H}+\mathrm{M})$ & 32689 & $1.2 \pm 0.1^{\mathrm{f}}$ & $0.0 \pm 0.01$ & 5 \\
\hline & $32717-32719$ & $48 \pm 10^{\mathrm{e}}$ & $0.5 \pm 0.02$ & $9(\mathrm{H}+\mathrm{M})$ & 33 306-33 308 & $0.8 \pm 0.1^{\mathrm{f}}$ & $0.5 \pm 0.02$ & 5 \\
\hline \multirow[t]{3}{*}{ XZ Dra } & (41 078-41 168) & $>67 \pm 3$ & $0.81 \pm 0.07$ & $11(\mathrm{M})$ & (40 494-41 133) & $0.99 \pm 0.03$ & $0.83 \pm 0.02$ & 11,6 \\
\hline & (41 078-41 168) & $61: \pm 5$ & $0.18 \pm 0.09$ & $11(\mathrm{M})$ & (40 494-41 133) & $0.93 \pm 0.03$ & $0.18 \pm 0.12$ & 11,6 \\
\hline & $38929-38940$ & $43 \pm 5$ & $0.6 \pm 0.08$ & $13(\mathrm{M})$ & (36410-39403) & $0.72 \pm 0.05$ & $0.6 \pm 0.05$ & 11 \\
\hline \multirow[t]{14}{*}{ RR Lyr } & 37825 & $>42$ & 0.81 & $7(\mathrm{M})$ & & $0.62:^{\mathrm{g}}$ & 0.81 & 7 \\
\hline & 37833 & $>63$ & 0.00 & $7(\mathrm{M})$ & 37833 & 0.965 & 0.00 & 7 \\
\hline & 37855 & 36 & 0.54 & $7(\mathrm{M})$ & 37855 & $>0.5$ & 0.54 & 7 \\
\hline & 37858 & $>39$ & 0.61 & $7(\mathrm{M})$ & & $0.5:^{\mathrm{g}}$ & 0.61 & 7 \\
\hline & 37859 & $>37$ & 0.64 & $7(\mathrm{M})$ & 37859 & $>0.465$ & 0.64 & 7 \\
\hline & 37863 & $>37$ & 0.74 & $7(\mathrm{M})$ & 37863 & 0.56 & 0.74 & 7 \\
\hline & 37867 & $>47$ & 0.84 & $7(\mathrm{M})$ & 37867 & 0.71 & 0.84 & 7 \\
\hline & 38215 & 45 & 0.36 & $7(\mathrm{M})$ & 38215 & 0.67 & 0.36 & 7 \\
\hline & 38219 & 47 & 0.46 & $7(\mathrm{M})$ & 38219 & 0.65 & 0.46 & 7 \\
\hline & 38240 & 54 & 0.97 & $7(\mathrm{M})$ & 38240 & 0.79 & 0.97 & 7 \\
\hline & 38244 & 52 & 0.07 & $7(\mathrm{M})$ & 38244 & 0.75 & 0.07 & 7 \\
\hline & 38596 & $\geq 47$ & 0.70 & $7(\mathrm{M})$ & 38596 & 0.74 & 0.70 & 7 \\
\hline & 38601 & $\geq 40$ & 0.82 & $7(\mathrm{M})$ & 38601 & $>0.59$ & 0.82 & 7 \\
\hline & $43384-43388$ & $45 \pm 2$ & $0.63 \pm 0.05$ & $12(\mathrm{M})$ & & $0.70:^{\mathrm{g}}$ & $0.63 \pm 0.05$ & 7,10 \\
\hline \multirow[t]{3}{*}{ RV UMa } & (36 624-37 056) & $74 \pm 8^{\mathrm{h}}$ & $0.07 \pm 0.07$ & $8(\mathrm{H})$ & (36 647-37 061) & $>1.38 \pm 0.02$ & $0.07 \pm 0.07$ & 8,3 \\
\hline & (36 624-37 056) & $67 \pm 10^{\mathrm{h}}$ & $0.85 \pm 0.05$ & $8(\mathrm{H})$ & $(36647-37061)$ & $1.30 \pm 0.02$ & $0.900 \pm 0.005$ & 8,3 \\
\hline & (36 624-37 056) & $40 \pm 8^{\mathrm{h}}$ & $0.36 \pm 0.04$ & $8(\mathrm{H})$ & $(36647-37061)$ & $0.72 \pm 0.02$ & $0.375 \pm 0.025$ & 8,3 \\
\hline
\end{tabular}

a JD-2 400 000. Parentheses mean that observations of the given $\Phi_{\mathrm{B}}$ were selected from this interval.

b Errors include estimates of systematic discrepancies.

c [1] Fitch et al. (1966), [2] Jones et al. (1988), [3] Kanyó (1976), [4] Kanyó (1986), [5] Muller (1953), [6] Penston (1973), [7] Preston et al. (1965), [8] PS67, [9] Struve \& Van Hoof (1949), [10] Szeidl et al. (1997), [11] Szeidl et al. (2001), [12] Wilson et al. (1989), [13] Woolley \& Aly (1966).

d Lines that the $v_{\text {rad }}$ data were derived from (H: Hydrogen, M: Metal) are indicated.

e Derived from Van Hoof's measurements, based on using more metallic lines than Struve's data.

f Transformed from white light amplitude; the $A_{V}$ at the same $\Phi_{\mathrm{B}}$ during the JD $2433412-2434309$ interval was the same.

g Estimated from the amplitudes during other Blazhko cycles according $\Phi_{B}$, and the measured magnitudes of maxima, considering the phase of the 4-year modulation.

h Radial velocities of $\mathrm{H}$ lines are transformed into metallic scale according to $A_{v_{\mathrm{rad}}}^{(\mathrm{Met})}=0.742 A_{v_{\mathrm{rad}}}^{(\mathrm{H})}$ (see Fig. 1).

Only radial velocity observations with sufficiently good phase coverage to measure the total amplitude could be utilized, provided that a conterporary photometric $V$ amplitude could also be reliably determined.

Table 1 lists the radial velocity measurements as well as the corresponding photometric amplitudes of Blazhko stars. Most of the columns are self explanatory. The Blazhko phases $\left(\Phi_{\mathrm{B}}\right)$ of the observations were calculated using the ephemerides given in Table 2 .

The radial velocity data are very heterogeneous, obtained by using instruments of different resolutions. A further inconsistency arises from the selection of spectral lines used in determining the radial velocity. The relation between the radial velocity and photometric amplitudes of pulsation defined by Liu (1991) was based on radial velocities of metallic lines, so we considered metallic line velocities if they were given separately from the results for hydrogen lines. In the case of RV UMa, however, only radial velocities of $\mathrm{H}$ lines were published in PS67. We attempted to transform these to match the metallic line radial velocities utilizing the extended observations of RR Lyr (Preston et al. 1965). The Balmer lines originate from higher regions of the atmosphere exhibiting a larger velocity gradient than shown by the photospheric metallic lines. It was found that radial velocities measured from $\mathrm{H}$ and metallic lines are strongly correlated, and with an appropriate linear transformation can be equalized within their error 


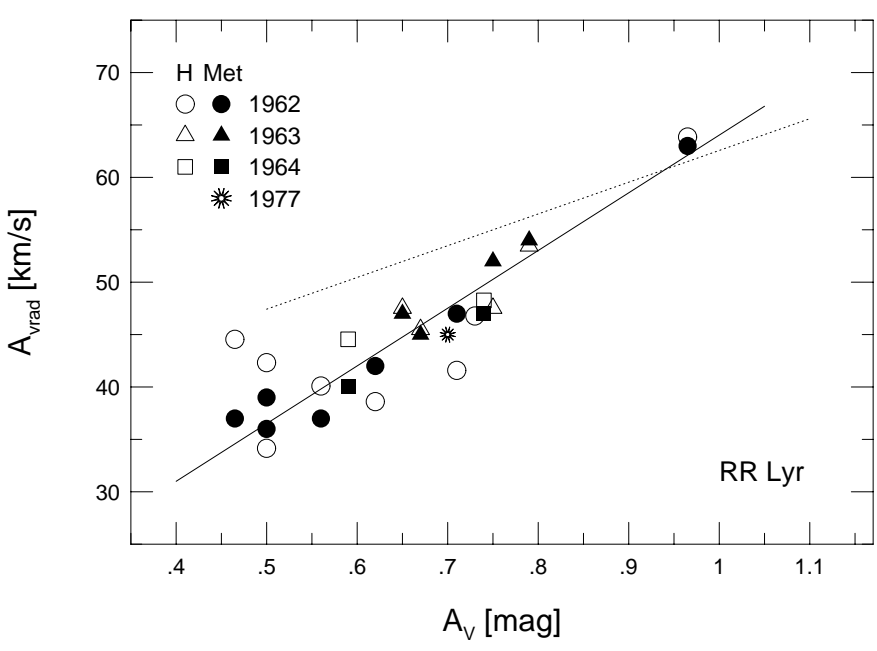

Fig. 1. Radial velocity amplitudes derived from metallic and hydrogen lines versus photometric $V$ amplitudes of RR Lyr according to the observations of Preston et al. (1965) and Wilson et al. (1989). Dotted line is for the location of single mode RR Lyrae stars (Liu 1991). The radial velocity amplitudes of $\mathrm{H}$ lines are divided by 1.347 in order to reach agreement with metallic line results. The 1963 and 1977 observations correspond to the minimum Blazhko activity phase of the 4-year cycle of RR Lyr, whilst the amplitude of the modulation varies within the 0.1-0.4 mag range.

ranges (see Fig. 1). Although this relation may differ from star to star, we transformed the PS67 radial velocities of RV UMa accordingly, to estimate its metallic line radial velocity amplitudes in different Blazhko phases.

Table 2. Blazhko modulation ephemerides.

\begin{tabular}{llc}
\hline \hline Name & Max Epoch [JD-2 400 000] & $P_{\mathrm{B}}[\mathrm{d}]$ \\
\hline RS Boo & 42786.0 & 532.0 \\
XZ Cyg & 17022.0 & 57.39 \\
XZ Dra & 36463.5 & 75.8 \\
& 40707.5 & 76.6 \\
AR Her & 49959.5 & 31.85 \\
RR Lyr & $34565 ; 38665 ; 41544 ; 42622 ; 43401 ; 44147$ & 40.80 \\
RV UMa & $36733.7 ; 37815.4$ & 90.785 \\
\hline
\end{tabular}

Ephemeris of XZ Cyg is taken from Smith (1975). Blazhko periods and maximum epochs for the other stars are determined from the collections of photometric data by Szeidl et al. (1997), Szeidl et al. (2001), Nagy (1998), and Kovács (1995) for RR Lyr, XZ Dra, RS Boo, and RV UMa, respectively. Different ephemerides for RR Lyr and $\mathrm{XZ}$ Dra take into account the phase shifts, and period changes of the modulation.

If there was no photometry simultaneous with the radial velocity observations, but the photometric amplitude for the same Blazhko phase could be reliably estimated from close (or distant) photometric observations, Table 1 lists these amplitudes.

Liu (1991) used the $A_{V}-A_{v_{\mathrm{p}}}$ relation, where $A_{v_{\mathrm{p}}}$ is the pulsational velocity amplitude. Since the transformation of $A_{v_{\text {rad }}}$ into $A_{v_{\mathrm{p}}}$ involves further uncertainties, we decided to use the directly measured radial velocity amplitudes. The regression of

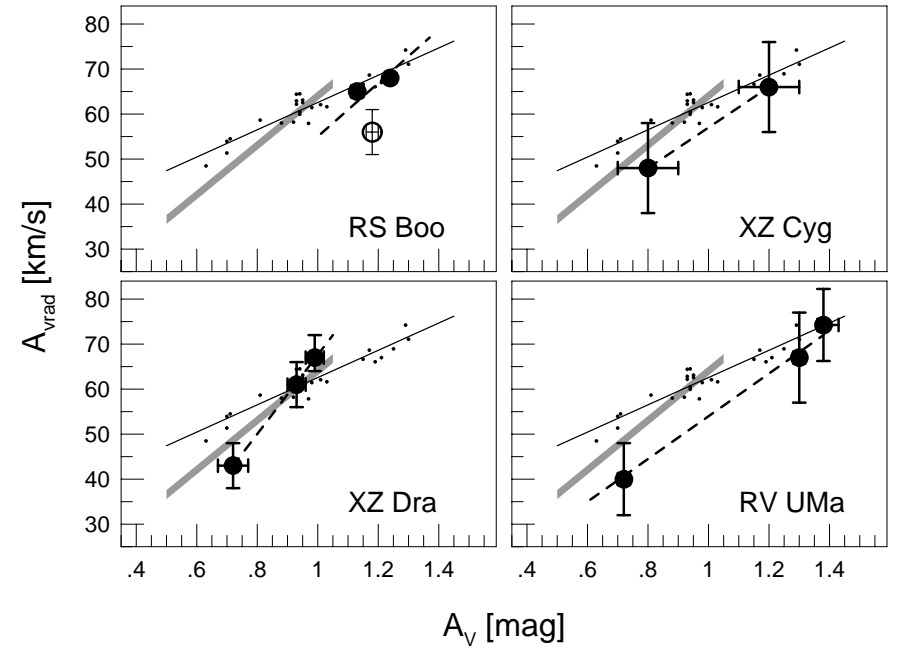

Fig. 2. Comparison of the radial velocity and photometric amplitudes of Blazhko type RR Lyrae stars with the sample of non Blazhko RRab stars (solid line fitted to small dots using the compilation of Liu 1991). The greyscale strip, shown in detail in Fig. 1, denotes the positions of RR Lyr. Blazhko stars lie along nearly parallel lines that match the area of normal RRab stars in the larger amplitude phases.

the $A_{V}-A_{v_{\text {rad }}}$ data of the sample used by Liu (1991, and references therein) has similar error statistics as the $A_{V}-A_{v_{\mathrm{p}}}$ dataset, justifying our choice.

Figure 2 shows the behaviour of 5 Blazhko variables in the $A_{V}-A_{v_{\text {rad }}}$ plane in different phases of their Blazhko cycles. In contrast to the early suggestion of PS67 the Blazhko stars seem to behave very similarly.

a) In the $A_{V}-A_{v_{\text {rad }}}$ plane they follow tracks with the same gradient within the limits of the observational uncertainties; these are steeper than the $A_{V}-A_{v_{\text {rad }}}$ relation defined by nonBlazhko variables. This means that the photometric amplitude of Blazhko stars in their smaller amplitude phase is larger than that of a normal RRab star with the same radial velocity amplitude.

b) The amplitude ratio of their photometric and radial velocity changes corresponds to that of non-Blazhko stars within 0.1-0.2 mag vicinity of their maximum photometric amplitudes.

\section{Light curve shapes}

The results shown in Sect. 2 hint that normal behaviour phases of Blazhko stars, if any, should be in the larger amplitude phase. To check the compatibility of Blazhko stars' light curves with that of unmodulated single mode RRab stars we utilize the Fourier decompositions of complete light curves of different Blazhko phases. The compatibility is verified if both the $D_{\mathrm{m}}$ criterion according to the formalism of Kovács \& Kanbur (1998) is valid, and $[\mathrm{Fe} / \mathrm{H}]$ calculated from the light curve parameters using the Jurcsik \& Kovács (1996) metallicity formula is in good agreement with direct spectroscopic measurements. Selection criteria are chosen at the $2 \sigma$ limit for $D_{\mathrm{m}}$ and \pm 0.15 dex for the difference between the measured and calculated metallicity values $\left(D_{\mathrm{m}}<2 ;|\Delta[\mathrm{Fe} / \mathrm{H}]|<0.15\right)$. 


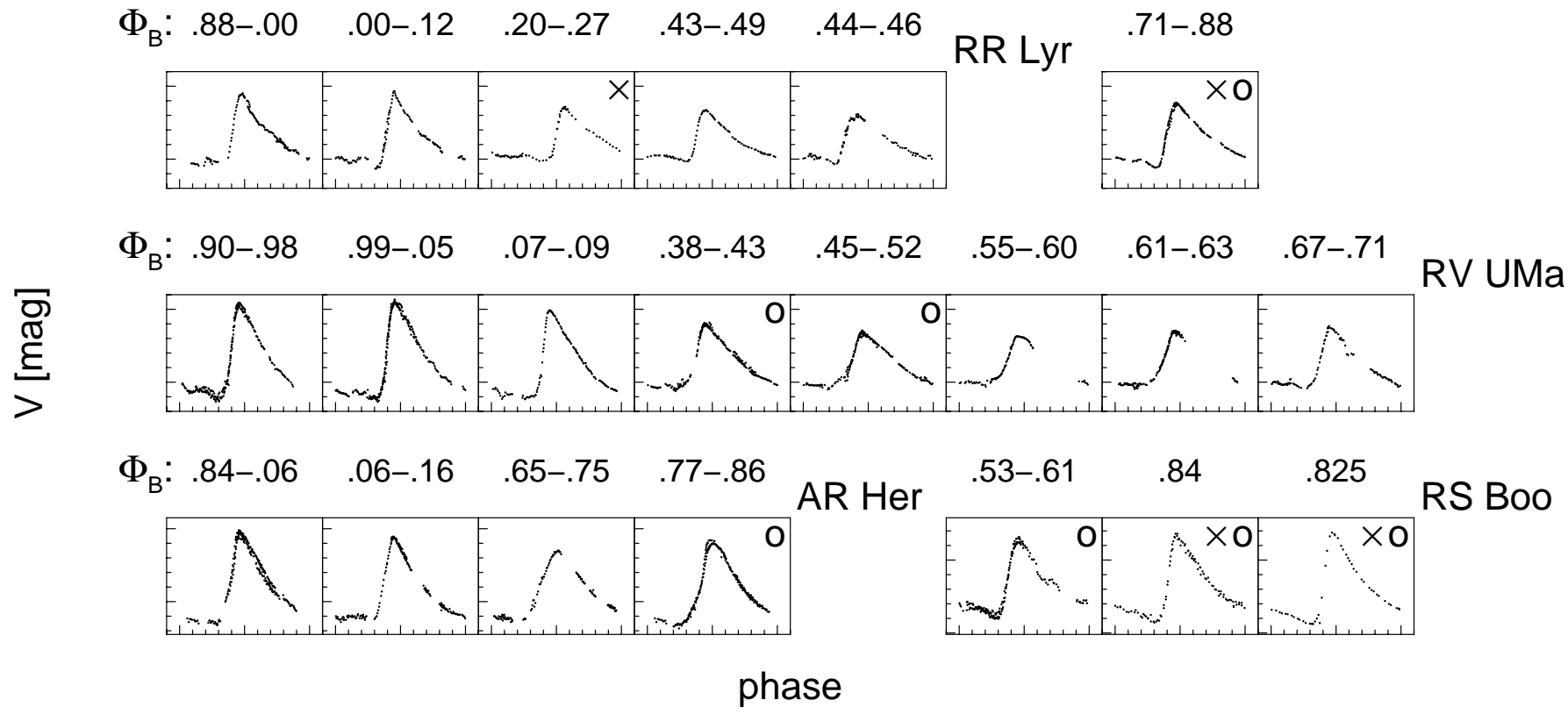

Fig. 3. Complete $V$ light curves of Blazhko variables during different phases of the modulation. Spacing of tick marks are 0.1 phase and 0.2 mag, respectively. Blazhko phases according to Table 2 are indicated in the top of the panels. For RR Lyr, data of Murnikova (1977, 1981), Fitch et al. (1966), Siegel (1982) and Hardie (1955) are used, observations of AR Her are taken from Smith et al. (1999), and the compilations of photoelectric observations of RV UMa and RS Boo (Kovács 1995; Nagy 1998) are utilized. Crosses (x) and circles (o) in the top right corners indicate $D_{\mathrm{m}}<2$ and $|\Delta[\mathrm{Fe} / \mathrm{H}]|<0.15$, respectively.

The observational strategy followed by observers of Blazhko type stars has concentrated mostly on the rising branch and the maxima, thus only very limited complete light curves of Blazhko stars are available. We have constructed 21 nearly complete light curves of four Blazhko variables with spectroscopically known metallicities. These light curves, shown in Fig. 3 are compiled from the observations of consecutive nights of the same Blazhko phases within a limited time interval, in order to evaluate the occurrence of long term changes of any type.

In Fig. 3 light curves with $D_{\mathrm{m}}<2$, and $|\Delta[\mathrm{Fe} / \mathrm{H}]|<0.15$ are denoted by circles and crosses in the top right corners, respectively. Only three light curves fulfil both requirements: two light curves of RS Boo and one of RR Lyr. This light curve of RR Lyr is, however, not representative for the large amplitude modulation, as these observations were obtained during the minima of the 4-year cycle (JD 2434553 -2 434560 ), when the amplitude of the modulation was smaller than 0.1 mag (Detre \& Szeidl 1973). The behaviour of RS Boo differs from other Blazhko type variables in many respects, it has an unusually short fundamental mode period, a very long modulation period and only reduced changes in its light curve shape. It is important to note that none of the large amplitude light curves of RV UMa fulfil both criteria, although from Fig. 2 it could be suspected that the large amplitude phase might be undistorted.

\section{Conclusions}

A combination of the $A_{V}-A_{v_{\text {rad }}}$ behaviour and the light curve shapes of Blazhko variables leads to the conclusion that for
Blazhko stars with large amplitude modulation there is no Blazhko phase which is compatible with that of a non-Blazhko RRab star. Although the amplitude ratio of the radial velocity and the light change match the normal mode pulsation during larger amplitude phases, none of these light curves has been proven to be undistorted.

Stars which seem to agree with non-Blazhko RRab stars in all the studied aspects are RR Lyrae in its low modulation amplitude phase of the 4-year cycle, and RS Boo whose amplitude modulation range is only about $0.15 \mathrm{mag}$.

This result means restriction for the theoretical explanation of the Blazhko phenomenon. Explanations that predict an undistorted light curve along any phase of a large amplitude modulation seem to be unrealistic.

Acknowledgements. We thank Géza Kovács and Andrea Nagy for providing us with the electronic compilations of RV UMa and RS Boo data, and to Andrew Wilkins for correcting the language of the manuscript. This research has made use of the SIMBAD database, operated at CDS Strasbourg, France. This work has been partly supported by OTKA grants T30954 and T30955.

\section{References}

Detre, L., \& Szeidl, B. 1973, in Variable Stars in Globular Clusters and Rel. Systems, ed. J. D. Fernie, ASSL, 36, 31

Fitch, W. S., Wiśniewski, W. Z., \& Johnson, H. L. 1966, Comm. Lunar Planet. Lab., 5, Part 2, 71

Hardie, R. H. 1955, ApJ, 122, 256

Jones, R. V., Carney, B. W., \& Latham, D. W. 1988, ApJ, 332, 206

Jurcsik, J., \& Kovács, G. 1996, A\&A, 312, 111

Kanyó, S. 1976, Comm. Konkoly Obs. Budapest, 7, 69

Kanyó, S. 1986, Comm. Konkoly Obs. Budapest, 10, 87 
Kovács, G. 1995, A\&A, 295, 693

Kovács, G., \& Kanbur, S. M. 1998, MNRAS, 295, 834

Kovács, G., \& Walker, A. 1999, ApJ, 512, 271

Kurochkin, N. E. 1959, Soviet Ast., 3, 676

Liu, T. 1991, PASP, 103, 205

Muller, A. B. 1953, Bull. Astron. Inst. Netherlands, 12, 11

Murnikova, V. P. 1977, Perem. Zvezdy Pril., 3, 221

Murnikova, V. P. 1981, Perem. Zvezdy Pril., 4, 1

Nagy, A. 1998, A\&A, 339, 440

Penston, M. J. 1973, MNRAS, 164, 133

Preston, G. W., Smak, J., \& Paczyński, B. 1965, ApJS, 12, 99

Preston, G. W., \& Spinrad, H. 1967, ApJ, 147, 1025 (PS67)

Siegel, M. J. 1982, PASP, 94, 122

Smith, H. A. 1975, PASP, 87, 465
Smith, H. A., Barnett, M., Silbermann, N. A., \& Gay, P. 1999, AJ, 118, 572

Struve, O., \& Van Hoof, A. 1949, ApJ, 109, 215

Szeidl, B. 1988, in Multimode Stellar Pulsations, ed. G. Kovács, L. Szabados, \& B. Szeidl (Budapest: Konkoly Obs. - Kultúra), 45

Szeidl, B., Guinan, E. F., Oláh, K., \& Szabados, L. 1997, Comm. Konkoly Obs. Budapest, 12, 99

Szeidl, B., Jurcsik J., Benkő, J. M., \& Bakos G. Á. 2001, Comm. Konkoly Obs. Budapest, 13, 101 (http://www.konkoly.hu/Mitteilungen/101.html)

Wilson, T. D., Carter, M. W., Barnes, T. G., Van Citters, G. W., \& Moffett, T. J. 1989, ApJS, 69, 951

Woolley, R., \& Aly, K. 1966, Roy. Obs. Bull., 114, 259 